\title{
Genetics of Endometrial Cancers
}

\section{Tsuyoshi Okuda, ${ }^{1}$ Akihiko Sekizawa, ${ }^{1}$ Yuditiya Purwosunu, ${ }^{1,2}$ Masaaki Nagatsuka, ${ }^{1}$ Miki Morioka, ${ }^{1}$ Masaki Hayashi, ${ }^{1}$ and Takashi Okai ${ }^{1}$}

\author{
${ }^{1}$ Department of Obstetrics and Gynecology, Showa University School of Medicine, 1-5-8 Hatanodai, Shinagawa-ku, \\ Tokyo 142-8666, Japan \\ ${ }^{2}$ Department of Obstetrics and Gynecology, University of Indonesia, Cipto Mangunkusumo National Hospital, Jakarta, Indonesia
}

Correspondence should be addressed to Tsuyoshi Okuda,t_okuda@med.showa-u.ac.jp

Received 22 October 2009; Revised 7 February 2010; Accepted 28 February 2010

Academic Editor: Enrique A. Hernández

Copyright ( $\odot 2010$ Tsuyoshi Okuda et al. This is an open access article distributed under the Creative Commons Attribution License, which permits unrestricted use, distribution, and reproduction in any medium, provided the original work is properly cited.

Endometrial cancers exhibit a different mechanism of tumorigenesis and progression depending on histopathological and clinical types. The most frequently altered gene in estrogen-dependent endometrioid endometrial carcinoma tumors is PTEN. Microsatellite instability is another important genetic event in this type of tumor. In contrast, p53 mutations or Her2/neu overexpression are more frequent in non-endometrioid tumors. On the other hand, it is possible that the clear cell type may arise from a unique pathway which appears similar to the ovarian clear cell carcinoma. K-ras mutations are detected in approximately $15 \%-30 \%$ of endometrioid carcinomas, are unrelated to the existence of endometrial hyperplasia. A $\beta$-catenin mutation was detected in about $20 \%$ of endometrioid carcinomas, but is rare in serous carcinoma. Telomere shortening is another important type of genomic instability observed in endometrial cancer. Only non-endometrioid endometrial carcinoma tumors were significantly associated with critical telomere shortening in the adjacent morphologically normal epithelium. Lynch syndrome, which is an autosomal dominantly inherited disorder of cancer susceptibility and is characterized by a MSH2/MSH6 protein complex deficiency, is associated with the development of non-endometrioid carcinomas.

\section{Introduction}

Endometrial cancer is the most common cancer of the female reproductive tract with 150,000 new cases diagnosed annually worldwide. Approximately 90\% of endometrial cancers are sporadic, and the remaining 10\% are hereditary. Bokhman have generally categorized endometrial cancer into two broad groups of tumors using both clinical and histopathological variables: estrogen-dependent endometrioid endometrial carcinomas (EECs), or type I, and nonendometrioid endometrial carcinomas (NEECs), or type II tumors (Table 1) [1]. It should be noted that this model is not strict, and only a minority of endometrial cancer may exhibit shared characteristics. For example, mixed serous and endometrioid tumors are being increasingly recognized. Approximately $70 \%$ to $80 \%$ of new cases are classified as EECs, and other $10 \%$ to $20 \%$ are designated as NEEC tumors [1]. EECs are strongly associated with the estrogen-related pathway and arise in association with unopposed estrogen stimulation [2]. In contrast, NEECs are unrelated to the estrogen pathways and arise in the background of atrophic endometrium [3]. EECs typically occur in premenopausal and younger postmenopausal women and are usually lowgrade and have a favorable outcome, whereas NEECs occur in older postmenopausal women. In addition, NEECs tend to predict a high tumor grade and poor patient prognosis $[4,5]$. The first pathway is associated with endometrioid histopathology, and the second is linked to the serous and clear cell subtypes. The precursors of these subtypes are known as atypical hyperplasia and endometrial intraepithelial carcinoma (EIC), respectively. Clear cell cancer, classified as an NEEC, is associated with atypical hyperplasia as well as EIC.

Recent reports suggest that histological differences may be associated with distinct molecular genetic alterations. Molecular genetic evidence indicates that endometrial 
carcinomas are likely to develop as the result of a multistep process of oncogenic activation and tumor suppressor inactivation (Table 2) [6].

\section{Gain-of-Function Genetic Events}

The genes implicated in the gain-of-function events are oncogenes. The important genes related to endometrial oncogenesis or progressions are the K-ras, B-raf, Her $2 / n e u, \beta$ catenin, AKT, and FGFR2 oncogenes.

2.1. K-ras and B-raf. K-ras proto-oncogene mutations are detected in approximately $10 \%-30 \%$ of endometrioid carcinomas [7]. K-ras mutations have been identified in endometrial hyperplasias, although at a lower frequency than in carcinomas [8-10]. According to these studies, the gain of the $K$-ras function may represent an early event in endometrioid-type tumorigenesis. During tumorigenesis, activated RAS is usually associated with enhanced proliferation, transformation, and cell survival. Conversely, $\mathrm{K}$-ras mutations occur with equal frequency in tumors with and without hyperplasia, and the epidemiologic results seem to suggest that $K$-ras activation is associated with malignant progression of endometrial tumors without the need for transition via hyperplasia [11]. In contrast to endometrioid carcinomas, $K$-ras mutations are extremely rare among serous and clear cell carcinomas $[12,13]$.

A correlation between colon cancer development and Ras/Raf point mutations in the MAP kinase pathway drives the malignant transformation of colon cancer. In contrast, only a few reports have shown $B$-raf mutations in patients with endometrial cancer. Feng et al. identified a $B$-raf mutation in $21 \%$ of patients with endometrial cancers and suggest that the mutation correlated with decreased hMLH1 expression [14]. In contrast, Salevesen et al. described a B-raf mutation in only $2 \%$ of endometrial cancers; and Kawaguchi et al. and Mizumoto et al. reported no mutation in the patients with endometrial cancer [15-17]. Therefore, a consensus about the role of $B$-raf mutation in the development of endometrial cancer has not yet been developed.

2.2. Her2/neu. Her2/neu (erbB2) is an oncogene that encodes a transmembrane receptor tyrosine kinase involved in cell signaling. Either the overexpression or gene amplification of Her2/neu proto-oncogene activates receptor and soluble tyrosine kinases. Her2/neu overexpression is detected in about $10 \%-20 \%$ of Grades 2 and 3 endometrioid carcinoma $[9,18,19]$. These studies suggest that Her2/neu overexpression in endometrioid carcinoma characterizes late progression and differentiation events. Her $2 /$ neu overexpression is detected in approximately $9 \%-30 \%$ of serous carcinomas [20]. Elucidation of the role of Her2/neu in these pathogenic tumor types, therefore, requires further study.

2.3. $\beta$-Catenin. $\beta$-catenin, a component of the E-cadherin family of proteins, is essential for cell differentiation and maintenance of normal tissue architecture, and plays an important role in signal transduction. $\beta$-catenin also acts as a downstream transcriptional activator in the Wnt signal transduction pathway. A $\beta$-catenin mutation results in the stabilization of proteins that are degradation resistant, thus resulting in cytoplasmic and nuclear $\beta$-catenin accumulation and constitutive target gene activity. The accumulation of $\beta$ catenin is demonstrated by immunohistochemistry. Several studies have analyzed endometrial cancers, showing that nuclear accumulation of $\beta$-catenin is significantly more common in endometrioid lesions (31\% to $47 \%$ ) compared to nonendometrioid histologies ( $0 \%$ to $3 \%$ ) [21]. In another report, $\beta$-catenin nuclear accumulation was more frequent in endometrial hyperplasias than in endometrial carcinoma samples, suggesting a $\beta$-catenin role in the early development of this tumor type [22]. In fact, alterations in $\beta$-catenin have been described in endometrial hyperplasia that contains squamous metaplasia or morules. Koul et al. found that all $\beta$-catenin mutated tumors were estrogen-receptor (ESR) positive and most were progesterone-receptor (PgR) positive, thus suggesting a dependence on estrogen stimulation during endometrial carcinogenesis [11]. In contrast, there is no correlation between $\beta$-catenin mutations and Microsatellite Instability (MI) or K-ras or PTEN mutations.

2.4. AKT. The phosphatidylinositol 3-kinase (PI3K) AKT pathway is activated in many human cancers and plays a key role in cell proliferation and survival. PIK3CA mutations frequently occur with other genetic alterations such as Her2/neu, K-ras, and PTEN in several types of tumors. Endometrial cancer is known to possess various genes alterations which activate the PI3K-AKT pathway. The frequency of mutations for PIK3CA in endometrial cancer is reported to be $28 \%$ [23]. However, Shoji et al. reported that AKT1 (E17K) mutations were detected in 2 out of 89 tissue samples and 0 out of 12 cell lines [24]. They suggested that AKT1 mutations might be mutually exclusive from other PI3KAKT activating alterations, although PIK3CA mutations frequently coexist with other gene aberrations. Additional mutations in AKT family members in endometrial cancers were reported in AKT2 (D399N, 426T, and 141T) and in AKT3 (E438D) [25]. Taken together, studies found that 5 out of 41 endometrial cancers have mutations in AKT family members at a frequency of approximately $12 \%$.

2.5. FGFR2. Alterations in the fibroblast growth factor receptor 2 (FGFR2) gene causes the receptors to become active, leading to cell proliferation. Byron et al. reported mutations in FGFR2 in 10\% of primary uterine tumor samples [26]. Mutations were observed in $16 \%$ of the endometrioid histology subtype tumors. In primary endometrioid endometrial cancers, FGFR2 and $\mathrm{K}$-ras mutations were mutually exclusive. Conversely, FGFR2 mutations were seen together with PTEN loss-of-function mutations. The authors also showed that endometrial cancer cell lines with activating FGFR2 mutations are selectively sensitive to the pan-FGFR inhibitor, PD173074 [27]. In addition, upregulation of FGF2 mRNA expression was observed in endometrial cancer specimens [28]. These data suggest that investigation of these 
TABLE 1: Clinical and pathological features of endometrial carcinoma.

\begin{tabular}{lll}
\hline & Type I (EEC) & Type II (NEEC) \\
\hline Age & Pre- and perimenopausal & Postmenopausal \\
Behavior & Stable & Progressive \\
Grade & Low & High \\
Hyperplasia-precursor & Present & Absent \\
Unopposed estrogen & Present & Absent \\
Myometrial invasion & Minimal & Deep \\
Specific Subtypes & Endometrioid carcinoma & Non-endometrioid carcinoma \\
Prevalence & $70-80 \%$ & $10-20 \%$ \\
Risk factors & Obesity, anovulation, nulliparity and exogenous estrogen exposure & In atropic endometrium \\
\hline
\end{tabular}

TABLE 2: Genetics features of endometrial carcinoma.

\begin{tabular}{lrr}
\hline & EEC & NEEC \\
\hline Gain-of Function & & \\
K-ras & $15-30 \%$ & $-5 \%$ \\
Her 2 neu & $10-20 \%$ & $9-30 \%$ \\
$\beta$-Catenin & $31-47 \%$ & $0-3 \%$ \\
Loss-of Function & & $10 \%$ \\
PTEN & $35-50 \%$ & $90 \%$ \\
P53 & $10-20 \%$ & $0-5 \%$ \\
Genomic instability (microsatellite) & $20-40 \%$ & \\
\hline
\end{tabular}

agents may be therapeutically beneficial for endometrial cancer patients.

\section{Loss-of-Function Genetic Events}

3.1. PTEN. Endometrial carcinomas are characterized by a variety of genetic alterations, but the most frequent alteration is in the PTEN gene. PTEN, located at chromosome 10q23, encodes a protein and lipid phosphatase which behaves as a tumor suppressor gene. PTEN inactivation is induced by mutations that lead to a loss of expression and is induced to a lesser extent by a loss of heterozygosity. The PTEN protein has both lipid and protein phosphatase activities, with each serving different functions. The lipid phosphatase activity of PTEN induces cell cycle arrest at the $\mathrm{G}_{1} / \mathrm{S}$ checkpoint. In addition, the upregulation of proapoptotic mechanisms involving AKT-dependent mechanisms is mediated through PTEN, as is the downregulation of antiapoptotic mechanisms through Bcl-2 [29-31]. PTEN further acts in opposition to PI3K to control levels of phosphorylated AKT $[23,32]$. A PI3K mutation is seen in $36 \%$ of endometrioid endometrial cancers and is common in tumors that also carry the PTEN mutation. The protein phosphatase activity of PTEN is involved in the inhibition of focal adhesion formation, cell spread, and migration, as well as the inhibition of growth-factor-stimulated MAPK signaling [33]. The PTEN gene, which acts as a tumor suppressor gene, is present in individuals and causes increased cancer susceptibility, including those with Cowden's syndrome. PTEN mutations are the most frequent genetic lesions in endometrial adenocarcinomas of the endometrioid subtype. PTEN mutations are reported in $25 \%-83 \%$ of tumors, more frequently in endometrioid carcinomas and microsatellite unstable tumors, and are, thus, the most frequent genetic alteration reported in cancers [34]. PTEN gene alterations are associated with metastatic behavior and advanced stage in other cancer types. In contrast, the loss of PTEN function is an early event in endometrial tumorigenesis. Several groups have described a concordance between MI status and PTEN mutations; the mutations occur in $60 \%-$ $86 \%$ of MI-positive endometrial carcinoma EEC cases, but only occur in $24 \%-35 \%$ of MI-negative tumors. Genetic alterations that account for PTEN protein inactivation include various mutations, a loss of heterozygosity $(\mathrm{LOH})$, or promoter hypermethylation, with mutations occurring the most frequently [30]. PTEN promoter methylation is observed in 19\% of cancers and is significantly associated with metastatic disease [35]. Kim et al. reported that PTEN and $K$-ras double-mutant mice ( ten $^{d / d} K$-ras ${ }^{G 12 D}$ ) exhibited dramatically accelerated endometrial cancer development compared to cancers formed from a single PTEN or K-ras gene mutation [36]. These results suggest a synergistic effect of dysregulation of the PTEN and $K$-ras signaling pathways during endometrial tumorigenesis.

3.2. P53. The $p 53$ gene is located on chromosome 17 and is important in preventing the propagation of cells with damaged DNA. $p 53$ mutations or TP53 overexpression is twice as frequent in tumors without hyperplasia (estrogen unrelated) than in those with hyperplasia (estrogen related) [11, 37]. This is consistent with other data in which the most striking genetic alteration, present in about $90 \%$ of serous carcinomas (estrogen-unrelated NEEC), is a $p 53$ mutation [38]. In other reports, statistically significant correlations were observed 
between p53 alterations and non-endometrioid histology type, high-grade tumors, and the absence of the progesterone receptor [39]. On the other hand, p53 genetic alterations were observed in $17 \%$ of endometrioid carcinomas, which were primarily Grade 3 [40]. The exact mechanisms causing this mutation are still not well characterized. In response to DNA damage, nuclear P53 accumulates and causes cell cycle arrest by inhibiting Cyclin D1 phosphorylation of the Rb gene and thereby promotes apoptosis. Therefore, mutated P53 results in a nonfunctional protein that accumulates in the cell and acts as a dominant negative inhibitor of wild-type P53, leading to propagation of aberrant cells. p53 mutations in endometrioid carcinoma are a late event during progression or differentiation. P53 alterations play a relatively minor role in clear cell type endometrial carcinoma in comparison to the serous type [41]. p53 mutations are also rarely observed in ovarian clear cell adenocarcinomas in comparison to endometrioid adenocarcinomas [42]. As a result, it is possible that the pathogenesis of clear cell carcinoma in the female genital tract arises from a unique pathway [43].

\section{Genomic Instability}

The most important types of genomic instability in endometrial cancers are MI and chromosomal aneuploidy. DNA mismatch repair (MMR) deficiency, detected as MI, is the most common molecular phenotype in endometrioid cancer, as PTEN tumor suppressor gene mutations. MI is seen in cancers (colonic, endometrial, and others) of patients with hereditary nonpolyposis colon cancer (HNPCC) and is also present in $28 \%$ of sporadic endometrioid cancers but is not present in serous cancers [40]. MI is distributed almost equally among the three histopathological tumor grades of endometrioid cancers. However, MI is rare in the clear cell type [44]. HNPCC patients with endometrial cancers have an inherited germline mutation in $\mathrm{MLH}-1, \mathrm{MSH}-2, \mathrm{MSH}-$ 6, or PMS-2, but endometrial cancer only develops after the instauration of a deletion or mutation in the contralateral MLH-1, MSH-2, MSH-6, or PMS-2 allele. Following this, the deficient MMR (MLH-1, MSH-2, MSH-6, or PMS-2) causes the acquisition of $\mathrm{MI}$ and the development of the tumor. Inactivation of the mismatch repair gene $M L H 1$ by methylation of the promoter seems to be the most frequent cause of MI in sporadic endometrioid carcinomas, followed by a loss of the expression of other two mismatch repair genes, the MSH2 and MSH6 genes. The mechanism for the inactivation of $\mathrm{MSH} 2$ is still not clear, as promoter methylation and mutations are rare. MSH6 inactivation is usually caused by a mutation.

Aneuploidy is frequent in serous cancers, and is uncommon in endometrioid cancer. When present, aneuploidy is exhibited predominantly by Grade 3 tumors $[45,46]$. These data suggest that a different type of genomic instability is associated with the different histopathological-type tumors. However, in some reports, no significant correlations were found to exist with either the $K$-ras or $p 53$ mutations $[7,11$, 47].
Telomeric attrition triggers genomic instability in certain cancer types. Both EEC and NEEC cells have short telomeres in endometrial cancer. However, only NEECs are significantly associated with critical telomere shortening compared to adjacent morphologically normal epithelium, thus suggesting that telomere shortening contributes to the initiation of NEECs but not EECs [48]. The authors also proposed a model in which telomere attrition gives rise to the initiation of NEECs and the progression of EECs.

\section{Genetics Events outside the Cancer Pathway}

Genetic variation acting either within or outside of the cancer cell may determine the outcome of interaction with exogenous or endogenous carcinogens. Endometrial stimulation by estrogens without the differentiating effects of the progestins is a primary etiologic factor associated with the development of endometrial hyperplasia and carcinoma [3]. There is evidence that estrogens and some of their metabolites are involved in the endometrial cancer pathogenesis. Estrogens and some of their derivatives are genotoxic and induce DNA damage, which if not removed could, thus, contribute to an increased risk of malignancy. Defects in the estrogen metabolism can result in defective apoptosis, DNA repair, and proliferation $[49,50]$. Estrogens mediate their effects via the estrogen receptors (ESRs), estrogen receptor alpha (ESR1) and estrogen receptor beta (ESR2), which activate its metabolic pathways. The polymorphisms of ESR1 and ESR2 suggest an association with an increasing risk of developing endometrial cancer [51]. Cytochrome P450 1B1 CYP1B1 is a constitutively expressed and inducible enzyme with a central role in the oxidative metabolism of a wide range of endogenous and exogenous compounds including many carcinogens $[52,53]$. Saini et al. reported that CYP1B1 depletion in endometrial cancer cells leads to decreased cellular proliferation and induced G0-G1 cell cycle arrest, thus suggesting that CYP1B1 inhibition in endometrial cancer cells could be a useful therapeutic approach [54]. Progesterone or its synthetic form has been used as a primary treatment or palliative treatment of advanced and recurrence endometrial cancer, because progesterone inhibits estrogeninduced endometrial proliferation. In addition, the loss of progesterone-mediated Wnt signaling inhibition in the endometrium plays a rate-limiting role in tumor onset and progression [55].

\section{Inherited Predisposition}

6.1. Lynch Syndrome. Lynch syndrome, or hereditary nonpolyposis colorectal cancer (HNPCC), is characterized by an increased risk for colorectal cancer. Endometrial cancer is the most common malignancy in patients with Lynch syndrome or HNPCC [56]. Lynch syndrome is caused by an inherited mutation in the MMR gene family, such as MLH1, MSH2, MSH6, PMS1, or PMS2 [57]. The age at diagnosis of Lynch syndrome associated endometrial cancer is approximately 2 decades younger than that for sporadic endometrial cancers [58]. Parc et al. demonstrated that $34 \%$ of young patients 
with endometrial cancer (median age 46) were associated with MI, $57 \%$ of the MI positive group showed an absence of $h M L H 1$ expression, $19 \%$ showed an absence of $h M L H 2$ expression, and $23.8 \%$ demonstrated a normal expression of both proteins, while $9.5 \%$ of all patients were diagnosed with Lynch syndrome [59]. In another report, the development of the latter tumors of Lynch syndrome is significantly associated with MSH2/MSH6 protein complex deficiency [60].

6.2. Familial Site-Specific Endometrial Carcinoma. The clustering of endometrial carcinoma alone, termed as familial site-specific endometrial carcinoma, may constitute a separate entity. Eight percent of this group have been reported to have germline MMR mutations [61]. This mutation rate is lower than that of Lynch syndrome with endometrial cancer patients, of whom 15\% show MMR mutations [62]. The difference in MMR, mutations, therefore suggests the existence of different genetic alteration pathways in familial site-specific endometrial carcinoma.

\section{Malignant Mixed Mullerian Tumors (MMMTs)}

Carcinosarcomas (malignant mixed mullerian tumors, or MMMTs) are currently excluded from uterine sarcoma and classified as metaplastic carcinoma, and many studies include these as NEECs [63]. However, endometrial carcinoma and MMMTs develop along distinctive molecular genetic pathways and exhibit different biological features. In MMMT, p53 alterations occur early, during progression, just prior to clonal expansion and acquisition of genetic diversity [64]. In addition, changes in the AKT/ $\beta$-catenin pathway may be essential for both the establishment and maintenance of phenotypic characteristics of MMMTs, playing key roles in the regulation of E-cadherin through transactivation of the Slug E-cadherin repressor gene [65]. Vaidya et al. reported that according to the discrepancy in survival the patients of MMMT should not be included in studies of endometrial cancers [66]. From this viewpoint, future studies will identify factors to classify these diseases.

\section{De-Differentiation of Endometrioid Tumors}

Mixed serous and endometrioid tumors have serous components that may be related to the "de-differentiation" of endometrioid tumors. This concept would explain the presence of overlapping EEC and NEEC features, both morphological and molecular in some tumors [67].

\section{Epigenetic Changes}

Aberrant $\mathrm{CpG}$ island hypermethylation in promoter regions occurs in many cancer-related genes, including those associated with cell cycle control, apoptosis, and DNA repair. Usually, unmethylated CpG islands become methylated, causing transcriptional silencing in cancer cells. Banno et al. reported that the frequencies of aberrant hypermethylation were $40.4 \%$ in $h M L H 1,22 \%$ in APC, $14 \%$ in E-cadherin, and $2.3 \%$ in $R A R-\beta$ in endometrial cancer specimens [68]. However, no aberrant DNA methylation was found in the p16 gene. Other genes inactivated by promoter hypermethylation in endometrial cancer include PgR [69], the cell cycle control genes 14-3-3 sigma [70], homeobox gene HOXA11, thrombospondin-2 gene (THBS2) [71], paternally expressed gene 3 (PEG3) [72], as well as the detoxifying enzyme glutathione S-transferase P1 (GSTP1) [73]. The impact of methylation on these genes in endometrial cancer development has not been well established. In endometrial cancers, differential DNA methylation patterns are detected in EICs and NEECs, suggesting divergent epigenetic backgrounds and unique tumorigenic pathways [74]. Promoter hypermethylation is a frequent event in EIC but not NEECs [75]. Many of the tumor suppressor pathways that are mutated in EIC can also be inactivated by hypermethylation.

\section{The Future}

The goal of screening endometrial cancers is to identify all patients who have a risk for developing this disease. Therefore clarification of the molecular and genetic mechanisms of development or progression of this disease is required. Understanding the genetic changes underlying cancer development or progression in the different histological subtypes is important for discovery of new targets for both diagnosis and therapy for individual patients.

\section{Acknowledgments}

This work was supported in part by Special Subsidies from the Subsidies for ordinary expenses of private schools from the Promotion and Mutual Aid Corporation for Private Schools of Japan, as well as research funding from the JAOG Ogyaa Donation Foundation (JODF) and the Takeda Science Foundation.

\section{References}

[1] J. V. Bokhman, "Two pathogenetic types of endometrial carcinoma," Gynecologic Oncology, vol. 15, no. 1, pp. 10-17, 1983.

[2] N. Potischman, R. N. Hoover, L. A. Brinton, et al., "Casecontrol study of endogenous steroid hormones and endometrial cancer," Journal of the National Cancer Institute, vol. 88, no. 16, pp. 1127-1135, 1996.

[3] M. E. Sherman, M. E. Bur, and R. J. Kurman, "p53 In endometrial cancer and its putative precursors: evidence for diverse pathways of tumorigenesis," Human Pathology, vol. 26, no. 11, pp. 1268-1274, 1995.

[4] S. G. Silverberg and O. B. Ioffe, "Pathology of cervical cancer," Cancer Journal, vol. 9, no. 5, pp. 335-347, 2003.

[5] P. Uharček, "Prognostic factors in endometrial carcinoma," Journal of Obstetrics and Gynaecology Research, vol. 34, no. 5, pp. 776-783, 2008.

[6] A. Berchuck and J. Boyd, "Molecular basis of endometrial cancer," Cancer, vol. 76, supplement 10, pp. 2034-2040, 1995. 
[7] I. B. Engelsen, L. A. Akslen, and H. B. Salvesen, "Biologic markers in endometrial cancer treatment," APMIS, vol. 117, no. 10, pp. 693-707, 2009.

[8] H. Sasaki, H. Nishii, H. Takahashi, et al., "Mutation of the $\mathrm{Ki}$-ras protooncogene in human endometrial hyperplasia and carcinoma," Cancer Research, vol. 53, no. 8, pp. 1906-1910, 1993.

[9] B. D. Duggan, J. C. Felix, L. I. Muderspach, J.-L. Tsao, and D. K. Shibata, "Early mutational activation of the c-Ki-ras oncogene in endometrial carcinoma," Cancer Research, vol. 54, no. 6, pp. 1604-1607, 1994.

[10] A. Doll, M. Abal, M. Rigau, et al., "Novel molecular profiles of endometrial cancer-new light through old windows," Journal of Steroid Biochemistry and Molecular Biology, vol. 108, no. 35, pp. 221-229, 2008.

[11] A. Koul, R. Willén, P.-O. Bendahl, M. Nilbert, and A. Borg, "Distinct sets of gene alterations in endometrial carcinoma implicate alternate modes of tumorigenesis," Cancer, vol. 94, no. 9, pp. 2369-2379, 2002.

[12] M. Esteller, A. García, J. M. Martínez-Palones, J. Xercavins, and J. Reventós, "The clinicopathological significance of KRAS point mutation and gene amplification in endometrial cancer," European Journal of Cancer, vol. 33, no. 10, pp. 15721577, 1997.

[13] A. Semczuk, H. Berbeć, M. Kostuch, M. Cybulski, J. Wojcierowski, and W. Baranowski, "K-ras gene point mutations in human endometrial carcinomas: correlation with clinicopathological features and patients' outcome," Journal of Cancer Research and Clinical Oncology, vol. 124, no. 12, pp. 695-700, 1998.

[14] Y.-Z. Feng, T. Shiozawa, T. Miyamoto, et al., "BRAF mutation in endometrial carcinoma and hyperplasia: correlation with KRAS and p53 mutations and mismatch repair protein expression," Clinical Cancer Research, vol. 11, no. 17, pp. 61336138, 2005.

[15] H. B. Salvesen, R. Kumar, I. Stefansson, et al., "Low frequency of BRAF and CDKN2A mutations in endometrial cancer," International Journal of Cancer, vol. 115, no. 6, pp. 930-934, 2005.

[16] M. Kawaguchi, M. Yanokura, K. Banno, et al., "Analysis of a correlation between the BRAF V600E mutation and abnormal DNA mismatch repair in patients with sporadic endometrial cancer," International Journal of Oncology, vol. 34, no. 6, pp. 1541-1547, 2009.

[17] Y. Mizumoto, S. Kyo, N. Mori, et al., "Activation of ERK1/2 occurs independently of KRAS or BRAF status in endometrial cancer and is associated with favorable prognosis," Cancer Science, vol. 98, no. 5, pp. 652-658, 2007.

[18] O. B. Ioffe, J. C. Papadimitriou, and C. B. Drachenberg, "Correlation of proliferation indices, apoptosis, and related oncogene expression (bcl-2 and c-erbB-2) and p53 in proliferative, hyperplastic, and malignant endometrium," Human Pathology, vol. 29, no. 10, pp. 1150-1159, 1998.

[19] J. A. Williams Jr., Z.-R. Wang, R. S. Parrish, L. J. Hazlett, S. T. Smith, and S. R. Young, "Fluorescence in situ hybridization analysis of HER-2/neu, c-myc, and p53 in endometrial cancer," Experimental and Molecular Pathology, vol. 67, no. 3, pp. 135143, 1999.

[20] B. M. Slomovitz, R. R. Broaddus, T. W. Burke, et al., "Her2/neu overexpression and amplification in uterine papillary serous carcinoma," Journal of Clinical Oncology, vol. 22, no. 15, pp. 3126-3132, 2004.
[21] G. Moreno-Bueno, D. Hardisson, C. Sánchez, et al., "Abnormalities of the APC/ $\beta$-catenin pathway in endometrial cancer," Oncogene, vol. 21, no. 52, pp. 7981-7990, 2002.

[22] H. Nei, T. Saito, H. Yamasaki, H. Mizumoto, E. Ito, and R. Kudo, "Nuclear localization of $\beta$-catenin in normal and carcinogenic endometrium," Molecular Carcinogenesis, vol. 25, no. 3, pp. 207-218, 1999.

[23] T. L. Yuan and L. C. Cantley, "PI3K pathway alterations in cancer: variations on a theme," Oncogene, vol. 27, no. 41, pp. 5497-5510, 2008.

[24] K. Shoji, K. Oda, S. Nakagawa, et al., "The oncogenic mutation in the pleckstrin homology domain of AKT1 in endometrial carcinomas," British Journal of Cancer, vol. 101, no. 1, pp. 145$148,2009$.

[25] A. Dutt, H. B. Salvesen, H. Greulich, W. R. Sellers, R. Beroukhim, and M. Meyerson, "Somatic mutations are present in all members of the AKT family in endometrial carcinoma," British Journal of Cancer, vol. 101, no. 7, pp. 12181219, 2009.

[26] S. A. Byron, M. G. Gartside, C. L. Wellens, et al., "Inhibition of activated fibroblast growth factor receptor 2 in endometrial cancer cells induces cell death despite PTEN abrogation," Cancer Research, vol. 68, no. 17, pp. 6902-6907, 2008.

[27] S. A. Byron and P. M. Pollock, "FGFR2 as a molecular target in endometrial cancer," Future Oncology, vol. 5, no. 1, pp. 27-32, 2009.

[28] G. Soufla, S. Sifakis, and D. A. Spandidos, "FGF2 transcript levels are positively correlated with EGF and IGF-1 in the malignant endometrium," Cancer Letters, vol. 259, no. 2, pp. 146-155, 2008.

[29] G. L. Mutter, "PTEN, a protean tumor suppressor," American Journal of Pathology, vol. 158, no. 6, pp. 1895-1898, 2001.

[30] G. L. Mutter, M.-C. Lin, J. T. Fitzgerald, et al., "Altered PTEN expression as a diagnostic marker for the earliest endometrial precancers," Journal of the National Cancer Institute, vol. 92, no. 11, pp. 924-930, 2000.

[31] K. Kurose, X.-P. Zhou, T. Araki, S. A. Cannistra, E. R. Maher, and C. Eng, "Frequent loss of PTEN expression is linked to elevated phosphorylated Akt levels, but not associated with p27 and cyclin D1 expression, in primary epithelial ovarian carcinomas," American Journal of Pathology, vol. 158, no. 6, pp. 2097-2106, 2001.

[32] M. C. Boruban, K. Altundag, G. S. Kilic, and J. Blankstein, "From endometrial hyperplasia to endometrial cancer: insight into the biology and possible medical preventive measures," European Journal of Cancer Prevention, vol. 17, no. 2, pp. 133138, 2008.

[33] G. L. Maxwell, J. I. Risinger, C. Gumbs, et al., "Mutation of the PTEN tumor suppressor gene in endometrial hyperplasias," Cancer Research, vol. 58, no. 12, pp. 2500-2503, 1998.

[34] N. Bansal, V. Yendluri, and R. M. Wenham, "The molecular biology of endometrial cancers and the implications for pathogenesis, classification, and targeted therapies," Cancer Control, vol. 16, no. 1, pp. 8-13, 2009.

[35] H. B. Salvesen, N. MacDonald, A. Ryan, et al., "PTEN methylation is associated with advanced stage and microsatellite instability in endometrial carcinoma," International Journal of Cancer, vol. 91, no. 1, pp. 22-26, 2001.

[36] T. H. Kim, J. Wang, K. Y. Lee, et al., "The synergistic effect of conditional Pten loss and oncogenic $K$-ras mutation on endometrial cancer development occurs via decreased progesterone receptor action," Journal of Oncology, vol. 2010, Article ID 139087, 9 pages, 2010. 
[37] T. Kaku, T. Kamura, T. Hirakawa, et al., "Endometrial carcinoma associated with hyperplasia-immunohistochemical study of angiogenesis and p53 expression," Gynecologic Oncology, vol. 72, no. 1, pp. 51-55, 1999.

[38] H. Tashiro, C. Isacson, R. Levine, R. J. Kurman, K. R. Cho, and L. Hedrick, "p53 gene mutations are common in uterine serous carcinoma and occur early in their pathogenesis," American Journal of Pathology, vol. 150, no. 1, pp. 177-185, 1997.

[39] E.-J. Lee, T.-J. Kim, D. S. Kim, et al., "p53 alteration independently predicts poor outcomes in patients with endometrial cancer: a clinicopathologic study of 131 cases and literature review," Gynecologic Oncology, vol. 116, no. 3, pp. 533-538, 2010.

[40] S. F. Lax, B. Kendall, H. Tashiro, R. J. C. Slebos, and L. H. Ellenson, "The frequency of p53, K-ras mutations, and microsatellite instability differs in uterine endometrioid and serous carcinoma: evidence of distinct molecular genetic pathways," Cancer, vol. 88, no. 4, pp. 814-824, 2000.

[41] S. F. Lax, E. S. Pizer, B. M. Ronnett, and R. J. Kurman, "Clear cell carcinoma of the endometrium is characterized by a distinctive profile of $\mathrm{p} 53, \mathrm{Ki}-67$, estrogen, and progesterone receptor expression," Human Pathology, vol. 29, no. 6, pp. 551$558,1998$.

[42] T. Okuda, J. Otsuka, A. Sekizawa, et al., "p53 mutations and overexpression affect prognosis of ovarian endometrioid cancer but not clear cell cancer," Gynecologic Oncology, vol. 88, no. 3, pp. 318-325, 2003.

[43] K. K. Zorn, T. Bonome, L. Gangi, et al., "Gene expression profiles of serous, endometrioid, and clear cell subtypes of ovarian and endometrial cancer," Clinical Cancer Research, vol. 11, no. 18, pp. 6422-6430, 2005.

[44] H.-J. An, S. Logani, C. Isacson, and L. H. Ellenson, "Molecular characterization of uterine clear cell carcinoma," Modern Pathology, vol. 17, no. 5, pp. 530-537, 2004.

[45] P. Rosenberg, S. Wingren, E. Simonsen, O. Stàl, B. Risberg, and B. Nordenskjöld, "Flow cytometric measurements of DNA index and S-phase on paraffin-embedded early stage endometrial cancer: an important prognostic indicator," Gynecologic Oncology, vol. 35, no. 1, pp. 50-54, 1989.

[46] B. Sorbe, B. Risberg, and B. Frankendal, "DNA ploidy, morphometry, and nuclear grade as prognostic factors in endometrial carcinoma," Gynecologic Oncology, vol. 38, no. 1, pp. 22-27, 1990.

[47] E. M. Swisher, S. Peiffer-Schneider, D. G. Mutch, et al., "Differences in patterns of TP53 and KRAS2 mutations in a large series of endometrial carcinomas with or without microsatellite instability," Cancer, vol. 85, no. 1, pp. 119-126, 1999.

[48] E. A. Akbay, C. M. Contreras, S. A. Perera, et al., "Differential roles of telomere attrition in type I and II endometrial carcinogenesis," American Journal of Pathology, vol. 173, no. 2, pp. 536-544, 2008.

[49] J. D. Yager and N. E. Davidson, "Estrogen carcinogenesis in breast cancer," The New England Journal of Medicine, vol. 354, no. 3, pp. 270-282, 2006.

[50] W. Yue, R. J. Santen, J.-P. Wang, et al., “Genotoxic metabolites of estradiol in breast: potential mechanism of estradiol induced carcinogenesis," Journal of Steroid Biochemistry and Molecular Biology, vol. 86, no. 3-5, pp. 477-486, 2003.

[51] K. A. Ashton, A. Proietto, G. Otton, et al., "Estrogen receptor polymorphisms and the risk of endometrial cancer," BJOG: An International Journal of Obstetrics and Gynaecology, vol. 116, no. 8, pp. 1053-1061, 2009.
[52] F. J. Gonzalez and H. V. Gelboin, "Role of human cytochromes P450 in the metabolic activation of chemical carcinogens and toxins," Drug Metabolism Reviews, vol. 26, no. 1-2, pp. 165183, 1994.

[53] F. P. Guengerich and T. Shimada, "Oxidation of toxic and carcinogenic chemicals by human cytochrome P-450 enzymes," Chemical Research in Toxicology, vol. 4, no. 4, pp. 391-407, 1991.

[54] S. Saini, H. Hirata, S. Majid, and R. Dahiya, "Functional significance of cytochrome P450 1B1 in endometrial carcinogenesis," Cancer Research, vol. 69, no. 17, pp. 7038-7045, 2009.

[55] Y. Wang, P. Hanifi-Moghaddam, E. E. Hanekamp, et al., "Progesterone inhibition of Wnt/ $\beta$-catenin signaling in normal endometrium and endometrial cancer," Clinical Cancer Research, vol. 15, no. 18, pp. 5784-5793, 2009.

[56] J. J. Koornstra, M. J. Mourits, R. H. Sijmons, A. M. Leliveld, H. Hollema, and J. H. Kleibeuker, "Management of extracolonic tumours in patients with Lynch syndrome," The Lancet Oncology, vol. 10, no. 4, pp. 400-408, 2009.

[57] P. Peltomäki, H. F. A. Vasen, M.-L. Bisgaard, et al., "Mutations predisposing to hereditary nonpolyposis colorectal cancer: database and results of a collaborative study. The International Collaborative Group on Hereditary Nonpolyposis Colorectal Cancer," Gastroenterology, vol. 113, no. 4, pp. 1146-1158, 1997.

[58] G. Marra and C. R. Boland, "Hereditary nonpolyposis colorectal cancer: the syndrome, the genes, and historical perspectives," Journal of the National Cancer Institute, vol. 87, no. 15, pp. 1114-1125, 1995.

[59] Y. R. Parc, K. C. Halling, L. J. Burgart, et al., "Microsatellite instability and hMLH1/hMSH2 expression in young endometrial carcinoma patients: associations with family history and histopathology," International Journal of Cancer, vol. 86, no. 1, pp. 60-66, 2000.

[60] P. Schweizer, A.-L. Moisio, S. A. Kuismanen, et al., "Lack of MSH2 and MSH6 characterizes endometrial but not colon carcinomas in hereditary nonpolyposis colorectal cancer," Cancer Research, vol. 61, no. 7, pp. 2813-2815, 2001.

[61] M. Ollikainen, W. M. Abdel-Rahman, A.-L. Moisio, et al., "Molecular analysis of familial endometrial carcinoma: a manifestation of hereditary nonpolyposis colorectal cancer or a separate syndrome?" Journal of Clinical Oncology, vol. 23, no. 21, pp. 4609-4616, 2005.

[62] Y. Hirai, K. Banno, M. Suzuki, et al., "Molecular epidemiological and mutational analysis of DNA mismatch repair (MMR) genes in endometrial cancer patients with HNPCC-associated familial predisposition to cancer," Cancer Science, vol. 99, no. 9, pp. 1715-1719, 2008.

[63] E. D’Angelo and J. Prat, "Uterine sarcomas: a review," Gynecologic Oncology, vol. 116, no. 1, pp. 131-139, 2010.

[64] S. F. Lax, "Molecular genetic changes in epithelial, stromal and mixed neoplasms of the endometrium," Pathology, vol. 39, no. 1, pp. 46-54, 2007.

[65] M. Saegusa, M. Hashimura, T. Kuwata, and I. Okayasu, "Requirement of the Akt/ $\beta$-catenin pathway for uterine carcinosarcoma genesis, modulating E-cadherin expression through the transactivation of slug," American Journal of Pathology, vol. 174, no. 6, pp. 2107-2115, 2009.

[66] A. P. Vaidya, N. S. Horowitz, E. Oliva, E. F. Halpern, and L. R. Duska, "Uterine malignant mixed mullerian tumors should not be included in studies of endometrial carcinoma," Gynecologic Oncology, vol. 103, no. 2, pp. 684-687, 2006. 
[67] C. S. Herrington, "Recent advances in molecular gynaecological pathology," Histopathology, vol. 55, no. 3, pp. 243-249, 2009.

[68] K. Banno, M. Yanokura, N. Susumu, et al., "Relationship of the aberrant DNA hypermethylation of cancer-related genes with carcinogenesis of endometrial cancer," Oncology Reports, vol. 16, no. 6, pp. 1189-1196, 2006.

[69] L. Ghabreau, J. P. Roux, A. Niveleau, et al., "Correlation between the DNA global methylation status and progesterone receptor expression in normal endometrium, endometrioid adenocarcinoma and precursors," Virchows Archiv, vol. 445, no. 2, pp. 129-134, 2004.

[70] P. Mhawech, A. Benz, C. Cerato, et al., "Downregulation of $14-3-3_{\sigma}$ in ovary, prostate and endometrial carcinomas is associated with CpG island methylation," Modern Pathology, vol. 18, no. 3, pp. 340-348, 2005.

[71] B. P. Whitcomb, D. G. Mutch, T.J. Herzog, J. S. Rader, R. K. Gibb, and P. J. Goodfellow, "Frequent HOXA11 and THBS2 promoter methylation, and a methylator phenotype in endometrial adenocarcinoma," Clinical Cancer Research, vol. 9, no. 6, pp. 2277-2287, 2003.

[72] S. C. Dowdy, B. S. Gostout, V. Shridhar, et al., "Biallelic methylation and silencing of paternally expressed gene 3 (PEG3) in gynecologic cancer cell lines," Gynecologic Oncology, vol. 99, no. 1, pp. 126-134, 2005.

[73] Q. K. Y. Chan, U.-S. Khoo, K. Y. K. Chan, et al., "Promoter methylation and differential expression of $\pi$-class glutathione S-transferase in endometrial carcinoma," Journal of Molecular Diagnostics, vol. 7, no. 1, pp. 8-16, 2005.

[74] X. C. Zhou, S. C. Dowdy, K. C. Podratz, and S.-W. Jiang, "Epigenetic considerations for endometrial cancer prevention, diagnosis and treatment," Gynecologic Oncology, vol. 107, no. 1, pp. 143-153, 2007.

[75] J. I. Risinger, G. L. Maxwell, A. Berchuck, and J. C. Barrett, "Promoter hypermethylation as an epigenetic component in type I and type II endometrial cancers," Annals of the New York Academy of Sciences, vol. 983, pp. 208-212, 2003. 


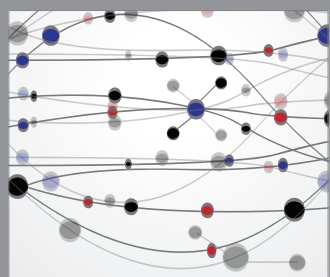

The Scientific World Journal
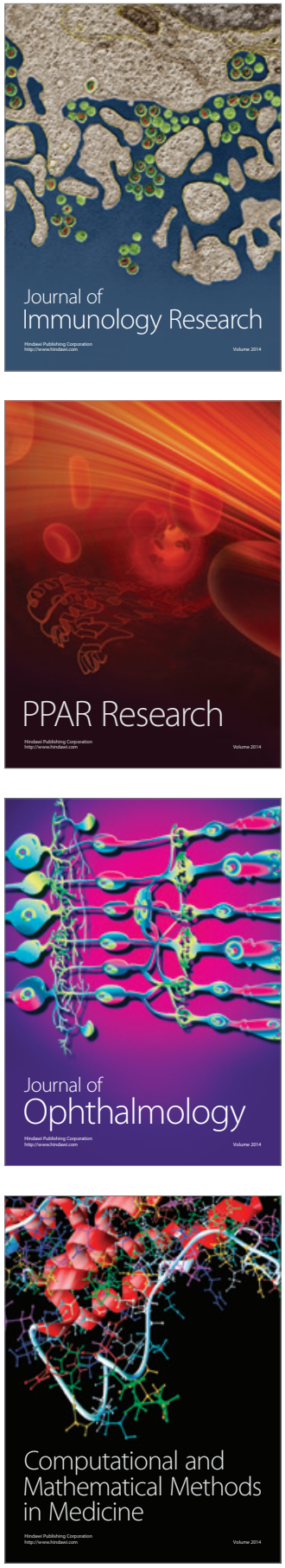

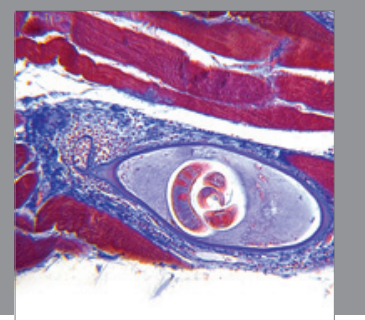

Gastroenterology

Research and Practice
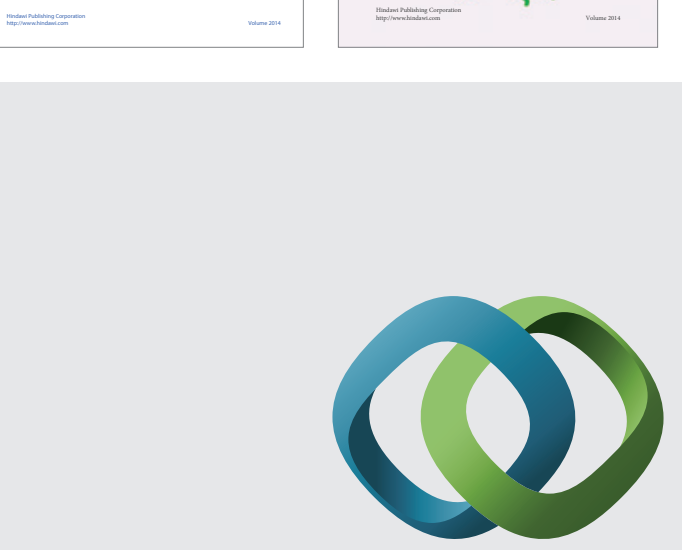

\section{Hindawi}

Submit your manuscripts at

http://www.hindawi.com
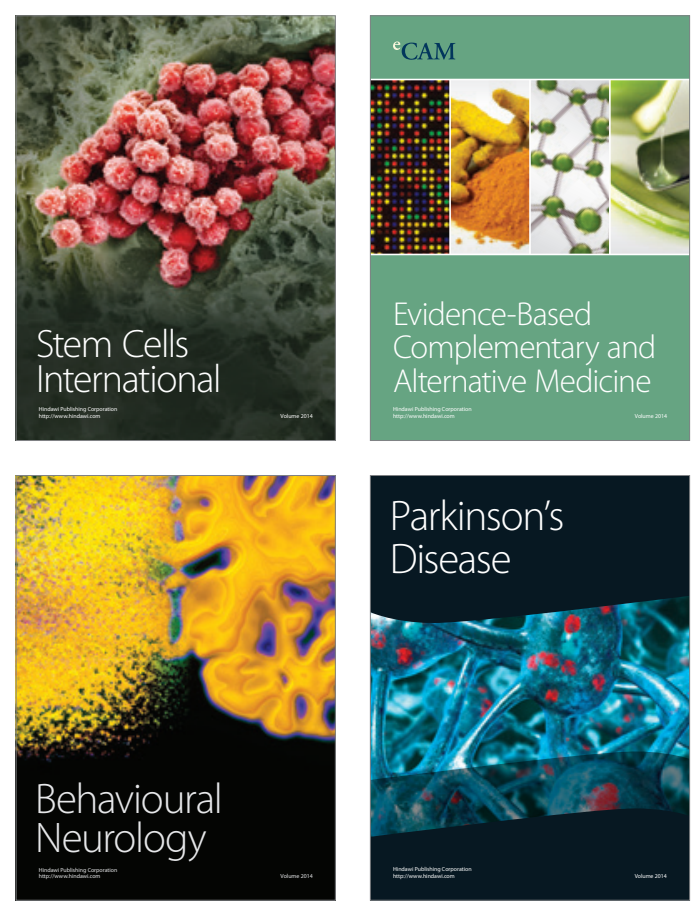

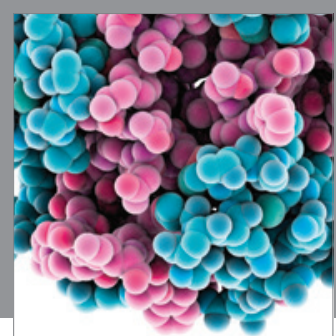

Journal of
Diabetes Research

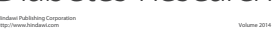

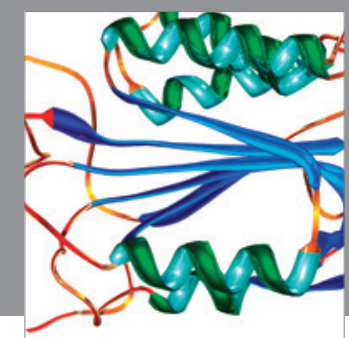

Disease Markers
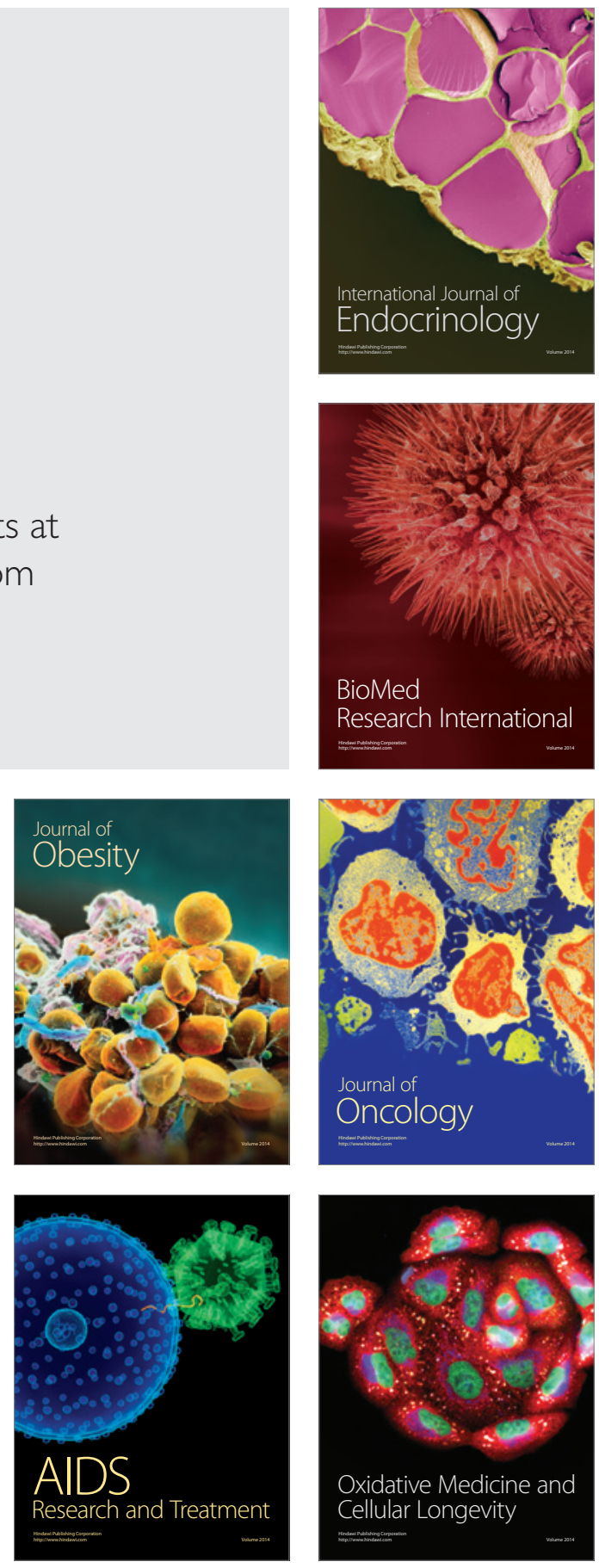